\title{
A Solution Quality Assessment Method for Swarm Intelligence Optimization Algorithms
}

\author{
Zhaojun Zhang, ${ }^{1}$ Gai-Ge Wang, ${ }^{2}$ Kuansheng Zou, ${ }^{1}$ and Jianhua Zhang' \\ ${ }^{1}$ School of Electrical Engineering and Automation, Jiangsu Normal University, Xuzhou, Jiangsu 221116, China \\ ${ }^{2}$ School of Computer Science and Technology, Jiangsu Normal University, Xuzhou, Jiangsu 221116, China \\ Correspondence should be addressed to Zhaojun Zhang; zzj921@gmail.com
}

Received 21 April 2014; Accepted 21 May 2014; Published 11 June 2014

Academic Editor: Xin-She Yang

Copyright (c) 2014 Zhaojun Zhang et al. This is an open access article distributed under the Creative Commons Attribution License, which permits unrestricted use, distribution, and reproduction in any medium, provided the original work is properly cited.

\begin{abstract}
Nowadays, swarm intelligence optimization has become an important optimization tool and wildly used in many fields of application. In contrast to many successful applications, the theoretical foundation is rather weak. Therefore, there are still many problems to be solved. One problem is how to quantify the performance of algorithm in finite time, that is, how to evaluate the solution quality got by algorithm for practical problems. It greatly limits the application in practical problems. A solution quality assessment method for intelligent optimization is proposed in this paper. It is an experimental analysis method based on the analysis of search space and characteristic of algorithm itself. Instead of "value performance," the "ordinal performance" is used as evaluation criteria in this method. The feasible solutions were clustered according to distance to divide solution samples into several parts. Then, solution space and "good enough" set can be decomposed based on the clustering results. Last, using relative knowledge of statistics, the evaluation result can be got. To validate the proposed method, some intelligent algorithms such as ant colony optimization (ACO), particle swarm optimization (PSO), and artificial fish swarm algorithm (AFS) were taken to solve traveling salesman problem. Computational results indicate the feasibility of proposed method.
\end{abstract}

\section{Introduction}

Swarm intelligence (SI) optimization [1] is a class of certain population-based metaheuristics which are inspired by the behavior of swarm of agents (i.e., living beings) interacting locally with each other and with their environment. SI is relatively new subfield of artificial intelligence. The behavior of every agent in SI is simple and does not have intelligence. But a number of simple agents through local rules are able to have the emergence of collective intelligence and come to intelligent solutions for complex problems. In recent years, SI has received widespread attention in research. Typical SI schemes include ant colony optimization (ACO) [2], particle swarm optimization (PSO) [3], artificial bee colony (ABC) [4], and artificial fish swarm algorithm (AFS) [5].

ACO is a class of optimization algorithms modeled on the foraging behavior of an ant colony. In ACO, a colony of artificial ants with the artificial pheromone trails and heuristic information are stochastic constructive heuristics that build better and better solutions by using and updating pheromone trail. New solutions are generated using a parameterized probabilistic model, the parameters of which are updated using previously generated solutions so as to direct the search towards promising areas of the solution space. The first ACO algorithm is ant system (AS) [6]. In the next years, many kinds of ACO algorithms have been developed to improve the performance of AS, such as ant colony system (ACS) [7], max-min ant system (MMAS) [8], and two-stage updating pheromone for invariant ant colony optimization algorithm (TSIACO) [9]. PSO is a metaheuristic search method that simulates the movements of a flock of birds which aim to find food. PSO optimizes a problem by having a population of candidate solutions, called particles, and moving these particles around in the search space according to simple mathematical formulae over the particle's position and velocity. Each particle's movement is influenced by its local best known position and also guided toward the best known positions in the search space, which are updated as 
better positions founded by other particles. The first PSO algorithm was introduced by Kennedy and Eberhart. ABC is an optimization algorithm based on the intelligent foraging behavior of honey bee swarm, proposed by Karaboga in 2005 . In the $\mathrm{ABC}$ model, the colony consists of three groups of bees: employed bees, onlookers, and scouts. It is assumed that there is only one artificial employed bee for each food source. Employed bees go to their food source and come back to hive and dance on this area. The employed bee whose food source has been abandoned becomes a scout and starts to search for finding a new food source. Onlookers watch the dances of employed bees and choose food sources depending on dances. The scout bee moves in the solution space to discover new food sources. SI has been applied to many applications problems, such as knapsack problems, scheduling problems, assignment problems, multiobjective optimization problem, and cluster analysis.

Although great progress has been achieved in application, there is also a basic question, which is how to quantify the goodness of the solution obtained in finite time, needed to be answered. We call it solution quality evaluation problem. At present, the existing researches focus on the solution "value performance," namely, the difference between the solution obtained by algorithm and the optimal solution of the problem. The general use of the method is ratio analysis, namely, ratio between solution obtained by algorithm and optimal solution. If the ratio is closer to 1 , it means that higher quality is obtained by algorithm and the algorithm is more effective. Competitive analysis [10] of online algorithm also can be employed. The drawback of two methods is that they need optimal solution of problem. There are some approximation methods used to estimate optimal for example, extreme value theory [11] and Lagrange's relaxation method [12], to get the solution value or bound to replace the optimal solution in practical problems. This analysis method generally requires strong theoretical basis of mathematic and strong math skills, and even it is difficult or impossible to give this kind of boundary for most of the problems. In addition to bias in the theoretical study of evaluation methods, some scholars pay more attention to the experimental analysis method. Hoos and Stützle [13] proposed to analyze the performance and behavior of stochastic local search algorithm by experimental analysis method. The performance of several existing particle swarm optimization algorithms was compared by using this method, and an improved particle swarm optimization algorithm was introduced according to the law in [14].

With development of the ordinal optimization (OO) theory [15], the research changes the angle to solution "ordinal performance" to evaluate solution quality of optimization method. Here the solution "ordinal performance" refers to the judgment about whether the solution is belonging to the good enough solution set. Shen et al. [16] used solution comparison between heuristic methods and uniform sampling to evaluate the solution. The evaluation criterion is alignment probability used in OO. As the extension of this work, author used the knowledge of hypothesis testing to develop it into a theory in [17]. In this paper, we proposed an experimental analysis method based on the analysis of search space and characteristic of algorithm itself to evaluate the solution quality for SI.

The rest of this paper is organized as follows: Section 2 reviews the basic idea of $\mathrm{OO}$ and indicates the difficulty of quantifying solution quality by analyzing the existing method. Section 3 describes our experimental analysis method detailed. Some simulation results are presented in Section 4 to show the feasibility of proposed method. Finally, Section 5 concludes the paper.

\section{Basics of Ordinal Performance}

The ordinal performance is concerned with whether the solution belongs to the good enough set. The evaluation criterion is alignment probability. The definition of good enough set and alignment probability is introduced in $\mathrm{OO}$. So, in this section, we briefly overview OO.

2.1. Brief Overview of $\mathrm{OO}$. OO was first introduced by Ho et al. in 1992 [15], which has become an important tool for optimizing discrete event dynamic system (DEDS). There are two basic ideas in OO. The first idea is ordinal comparison; that is, "order" is easier to ascertain than "value." The second idea is goal softening. Instead of only caring about optimal solution, OO is willing to settle for the "good enough" solution.

In $\mathrm{OO}, \Theta$ is the search space and satisfies $|\Theta|=N$. The "good enough" set $G$ is defined as the top- $g$ of the search space $\Theta$ or top $p \%$ of the search space $\Theta$. It satisfies $|G|=g$. Selected set $S$ is selected by rule and satisfies $|S|=s$. OO can guarantee that $S$ contains top-g solutions of the search space with a high probability. It is called alignment probability in $\mathrm{OO}$ and denoted by $P_{\mathrm{AP}}$.

2.2. Ordinal Performance. The research of solution quality evaluation method transfers from the value performance to the ordinal performance, after the definition of the good enough set, selected set, and alignment probability introduced. Based on this knowledge, Shen et al. [17] proposed evaluation method, called ordinal optimization ruler (OO ruler), using the related knowledge of hypothesis testing. So we can use $\mathrm{OO}$ ruler to qualify the ordinal performance of solution. One of the intuitive understandings of OO ruler is that uniform samples are taken out from the whole search space and evaluated with a crude but computationally easy model when applying OO. After ordering via the crude performance estimates, the lined-up uniform samples can be seen as an approximate ruler. By comparing the heuristic design with such a ruler, we can quantify the heuristic design, just as we measure the length of an object with a ruler. If the OO ruler gets from all the solutions, it is an accurate ruler. But this is obviously an ideal situation for practical problems. It is proved that approximate OO ruler is also effective.

Theorem 1 (see [17]). If the $k$ solution obtained by optimization algorithm is better than $t$ solution of selected set obtained by uniform sampling, we can judge that the $k$ solution belongs to the top $p \%$ of the search space $\Theta$ at least. And the type II 
error probability is not larger than $\beta_{0}$. The relation between $s$, $\beta_{0}, t$, and $p \%$ is determined by

$$
\sum_{j=0}^{t-1}\left(\begin{array}{l}
s \\
j
\end{array}\right)(p \%)^{j}(1-p \%)^{s-j} \leq \beta_{0}
$$

where $\left(\begin{array}{c}s \\ j\end{array}\right)$ represents the number of different choices of $s$ designed out of $j$ distinguished ones.

In the case of given parameters of $s$ and $\beta_{0}$, we can get relation between t and $p \%$ through the list method.

For an arbitrary solution obtained by heuristic algorithm, we only need to compare it whether satisfies the conditions of Theorem 1, then we can make the corresponding judgment, so as to realize the evaluation ordinal performance of solution. But $\mathrm{OO}$ ruler has a premise. To get $\mathrm{OO}$ ruler, uniform sampling for search space is needed. It is also prerequisite for OO. The so-called uniform sampling refers to the same probability of getting arbitrary solution. It is also the reason why the uniform sampling can provide quantitative reference. But, for some problems, it is difficult to achieve uniform sampling, and thus it will not be able to get $\mathrm{OO}$ ruler. In addition, the price of getting $\mathrm{OO}$ ruler for huge solution space is very high. These two problems limit the application of $\mathrm{OO}$ ruler in solution evaluation. However, the introduction of ordinal performance has great inspiration for the research of solution quality evaluation for SI.

\section{The Framework of Assessment Method}

In this section, we take traveling salesman problem (TSP) as an example to describe experimental analysis method of solution quality evaluation.

3.1. Sample Characteristics of SI. For SI, the feature of the algorithm itself determines that the sampling method in the search space is not uniform. Especially by the partial reinforcement effect, it makes the algorithm more and more concentrated in certain regions. So it is not suitable for evaluating method directly using $\mathrm{OO}$ ruler. In addition, the algorithm produces a large number of feasible solutions. The feasible solution contains the search characteristics of some algorithms and the distribution of the solution space. To obtain the hidden information and its rational utilization through some analysis methods, we need to do some research. It plays an important role in the research of qualtiy evaluation and improving the algorithm performance.

3.2. The Framework of Assessment Method. Based on the above analysis, this paper presents a general framework of the quality evaluation method for SI. The framework contains three procedures. First, to get some internal approximate uniform subclass, using cluster method, the solution samples (corresponding to selected subset of $\mathrm{OO}$ ) were homogeneous processing. Second, discrete probability distribution solution samples of each subclass and the scale relationship of the subclass are estimated in the fitness space. Based on the characteristics of the subclass, the presupposition ratio of the good enough set is distributed to each subclass. Last, alignment probability is calculated according to the model of solution quality evaluation, so as to complete the evaluation of the solution quality.

3.2.1. Uniform Clustering for Nonuniform Samples. According to the characteristics of discrete space, uniform clustering of samples is that obtaining probability of solution is approximating same. Compared with the continuous space, clustering is very different from discrete space. General discrete spatial distance features are defined with the question, and not as the continuous space as a distance to define general way. This makes clustering method based on grid no longer applicable, which is used in continuous space such as density clustering and clustering method based on grid. And the huge solution sample set also limits the use of some special clustering method. Therefore, we need to design a suitable and efficient clustering algorithm based on demand.

Approximate sampling probability is the purpose of clustering. The approximate sampling probability here refers to the neighbor characteristics (including the distance and number of nearest neighbors) consistent approximation. A feasible method for TSP is to calculate the distance between all solution samples. Then clustering is done according to the nearest neighbor statistical feature of each sample distance. But it is only applicable to the small size of the solution sample. Another possible method is that the clustering centers are selected from the best solutions. The distance is calculated between each feasible solution and the cluster center. Then the solution samples are clustered according to the distance. The calculation complexity of this algorithm is low. It is more suitable for clustering large scale solution samples. In the next section, we use this clustering method.

3.2.2. The "Good Enough" Set Decomposition. The solution alignment probability is calculated using a priori ratio of the good enough set (the ration between the good enough set and search space) in OO. The ratio of each kind of the good enough sets is needed to know after clustering. The prior ratio requires decomposing prior ratio of each class. This decomposition has a certain relationship with each class distribution of samples and the class size. Therefore, the distribution characteristics of solution in the fitness value, as well as proportional relation of class size, are needed to estimate.

Estimation of distribution of solution in the fitness value is problem of one-dimensional distribution sequence estimation. The purpose of distribution estimation is to obtain the good enough set distribution. If the fitness value is arranged according to the order from small to large, ordered performance curve (OPC) can be obtained. For the minimization problem, the good enough set is in the first half of the OPC. To obtain a true estimation of the good enough set, you need to consider the types of OPC.

3.2.3. Ordinal Performance Estimation. The original search space after clustering is divided into $l$ approximate uniform partition. Search space $\Theta_{k}$, the good enough set $G_{i}$, and 
Step 1. Delete the same solution of $S$ according to distance, and denote the new set by $S 1$;

Step 2. Find the best solution from the set $S 1$, and denote $s^{0}$;

Step 3. Calculate the distance between the solution of $S 1$ and $s^{0}$, divide the set $S 1$ into some subset $S_{i}$ according to distance, and let $\left|S_{i}\right|=s_{i}$;

Step 4. Distribute the good enough solution set $G$;

Step 4.1. Get $M$ solutions from $S 1$ according to the order of fitness;

Step 4.2. Delete the infeasible and same solutions, and get $M 1$ solutions;

Step 4.3. Assemble $M 1$ solutions and $S 1$ solutions, and get $g$ solutions by ordering fitness;

Step 4.4. Calculate the distance between $g$ solutions and $s^{0}$, and count the number of $g_{i}$ according to the distance;

Step 5. Calculate the alignment probability $P_{\mathrm{AP}}$ by (4)

Algorithm 1: The main steps of assessment method.

selected set $S_{i}$ of each partition and search space $\Theta$, good enough set $G$, and selected set $S$ of the original search space have the following correspondence in the collection and base:

$$
\begin{aligned}
& \Theta_{1} \cup \Theta_{2} \cup \cdots \cup \Theta_{l}=\Theta, \\
& G_{1} \cup G_{2} \cup \cdots \cup G_{l}=G, \\
& S_{1} \cup S_{2} \cup \cdots \cup S_{l}=S, \\
& N_{1}+N_{2}+\cdots+N_{l}=N, \\
& N \equiv|\Theta|, \quad N_{i} \equiv\left|\Theta_{i}\right|, \quad \forall i=1, \ldots, l, \\
& g_{1}+g_{2}+\cdots+g_{l}=g, \\
& g \equiv|G|, \quad g_{i} \equiv\left|G_{i}\right|, \quad \forall i=1, \ldots, l, \\
& s_{1}+s_{2}+\cdots+s_{l}=s, \\
& s \equiv|S|, \quad s_{i} \equiv\left|S_{i}\right|, \quad \forall i=1, \ldots, l,
\end{aligned}
$$

where $|\cdot|$ is the base of set .

Since the probability of any feasible solution pumped into each subclass is the same, for a sampling result $\theta_{i}$ has

$$
P\left(\theta_{s}=\theta_{i}\right)=\frac{1}{N_{i}}, \quad \forall \theta_{i} \in \Theta_{i} .
$$

In this paper, we only concern the selected set whether has at least one solution in good enough set. So we can draw the following conclusions:

$$
P_{\mathrm{AP}}\{|S \cap G| \geq 1\}=1-\prod_{i=1}^{l}\left(1-p_{i} \%\right)^{s_{i}} .
$$

3.2.4. Procedures of Assessment Method. The main steps to get the evaluation method by the above analysis are described in Algorithm 1.

\section{Experimental Results and Analysis}

In this section, we take the Hopfield 10-city problem, which is also used in [17], as the example to demonstrate our experimental analysis method. The coordinates of the 10 cities are
$\{(0.4000,0.4439) ;(0.2439,0.1463) ;(0.1707,0.2293) ;(0.2293$, $0.7610)$; $(0.5171,0.9414) ;(0.8732,0.6536) ;(0.6878,0.5219)$; $(0.8488,0.3609) ;(0.6683,0.2536) ;(0.6195,0.2634)\}$. There is $(10-1) !=362880$ solutions in the search space. The best path is $[1,4,5,6,7,8,9,10,2,3]$ or $[1,3,2,10,9,8,7,6,5,4]$. Here we define $|G|=0.005 \mathrm{~N}$. We use two groups of experimental simulation to demonstrate effectiveness of proposed method, where $P_{\mathrm{AP}}$ is alignment probability. Statistics value represents the alignment probability by our methods. Computational value is the alignment probability, and the error represents the difference of two alignment probabilities.

4.1. Evaluation Index. Alignment probability is a measure of whether optimal solution belongs to the good enough set. It is a probability value. Therefore, studying this value has little significance in one experiment. It is needed to do many experiments to study the statistical laws. So, each kind of experiment independently does $K$ times. If the optimal of $i$ time belongs to the good enough set, let $s_{i}=1$; otherwise $s_{i}=0$. Let $P_{g}$ be statistical frequency. Then, for $K$ times experiment, we have

$$
P_{g}=\frac{\sum_{i=1}^{r} s_{i}}{r}, \quad r=1,2, \ldots, K .
$$

From (5), the following can be seen, when $K$ tends to infinity:

$$
\lim _{K \rightarrow+\infty} P_{g}=P_{g}^{\prime}
$$

where $P_{g}^{\prime}$ is the alignment probability value, but it is generally difficult to obtain. In general, we only need to compute the $P_{g}$ value which may be tested experimentally.

Let $\bar{P}_{A}(r)$ be the alignment probability in an experiment by the evaluation method; $P_{A}$ is average value of $\bar{P}_{A}(r)$. Consider

$$
P_{A}=\frac{\sum_{i=1}^{r} \bar{P}_{A}(r)}{r}, \quad r=1,2, \ldots, K .
$$

Let $e_{r}$ be the absolute value of error of $P_{g}$ and $P_{A}$; that is,

$$
e_{r}=\left|P_{A}-P_{g}\right| \text {. }
$$




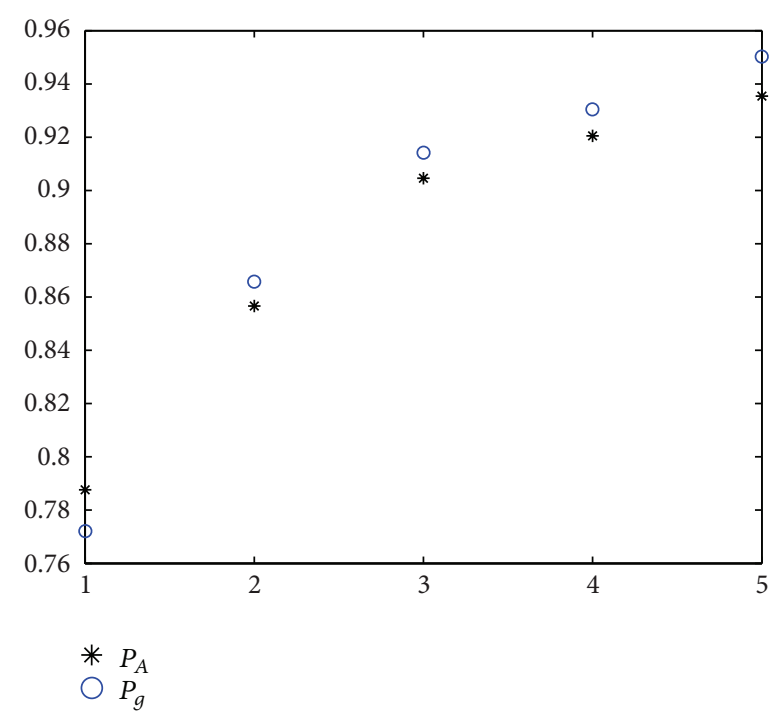

FIGURE 1: Comparison of two probabilities with ratio $\geq 1$.

In the following experiments, we are using $e_{r}$ as the standard evaluation index.

\subsection{Ordinal Performance Evaluation of Nonuniform Sampling.}

The solution space is sorted according to the fitness values and gets the whole solution space of the sample set, denoted by $\Omega$. We deliberately partition the search space into the same two parts $\Omega_{1}$ and $\Omega_{2}$. Then we sample, respectively, in parts $\Omega_{1}$ and $\Omega_{2}$, respectively. Times are denoted by $n_{1}$ and $n_{2}$. Then the total number of samplings is $n$. Then

$$
\begin{aligned}
& \frac{n_{1}}{n_{2}}=\text { ratio, } \\
& n_{1}+n_{2}=n .
\end{aligned}
$$

Let $K=5000$ and $n=3000$. Because the value of ration can be divided into two cases. One is no less than 1, and the other is less than 1 . So, the following points are discussed.

4.2.1. Ratio $\geq 1$. This case illustrates the sampling times in area $\Omega_{1}$ more than in area $\Omega_{2}$, and the good enough set is in area $\Omega_{1}$. The experiment results can be seen in Figures 1 and 2 . The abscissa is value of ratio. The values from left to right, respectively, are 1, 2, 5, 10, and 100. In Figure 1, we can see that, with the increasing value of ratio, the sampling point in area $\Omega_{1}$ is increasing. The probability of obtaining the good enough solution increases as the good enough set is in area $\Omega_{1}$. In addition, except for the case of ratio $=1, P_{A}$ is slightly higher than $P_{g}$. The rest of $P_{A}$ are lower than $P_{g}$. The error of two probabilities seen from Figure 2 is lower and no more than $2 \%$ generally.

4.2.2. Ratio $<1$. This case illustrates the sampling times in area $\Omega_{2}$ more than in area $\Omega_{1}$. The experiment results can be seen in Figures 3 and 4 . The abscissa is value of ratio. The values from left to right, respectively, are $0.01,0.1,0.2,0.5$, and

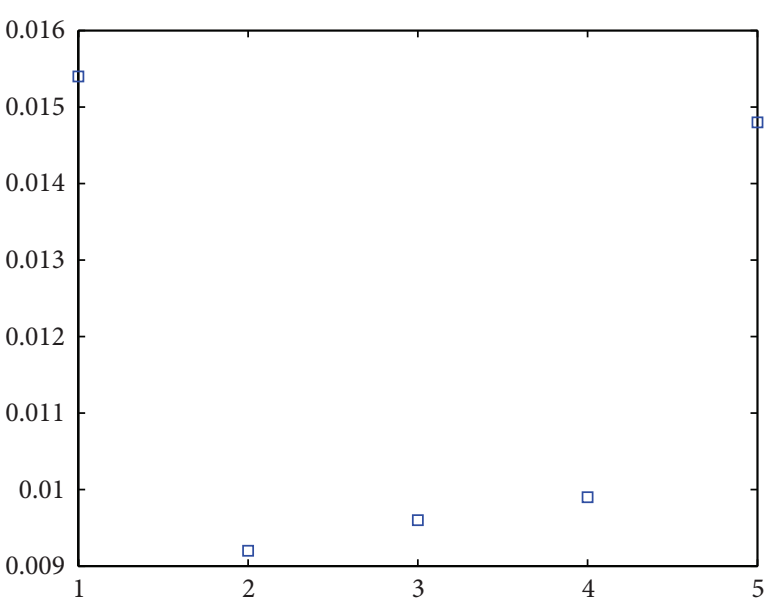

Figure 2: Error of two probabilities with ratio $\geq 1$.

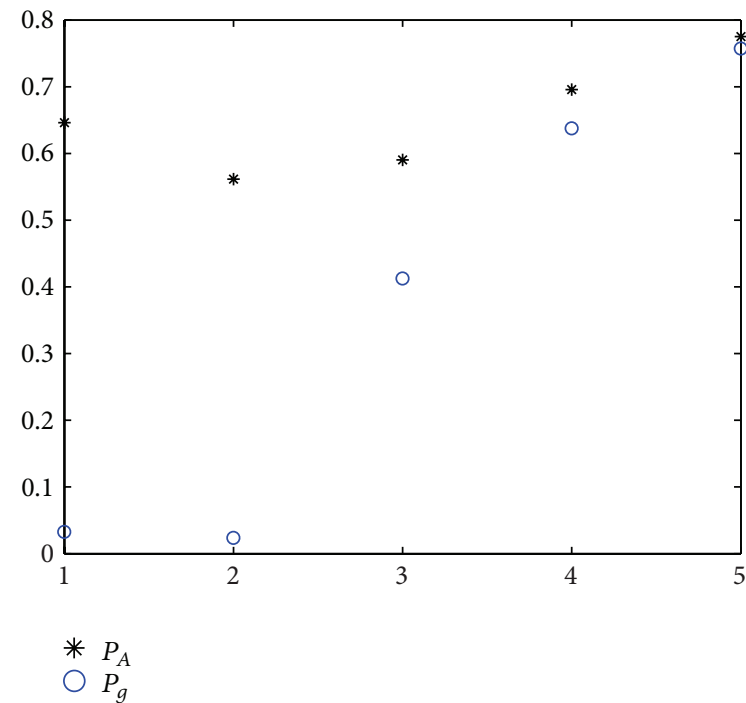

FIgURE 3: Comparison of two probabilities with ratio $<1$.

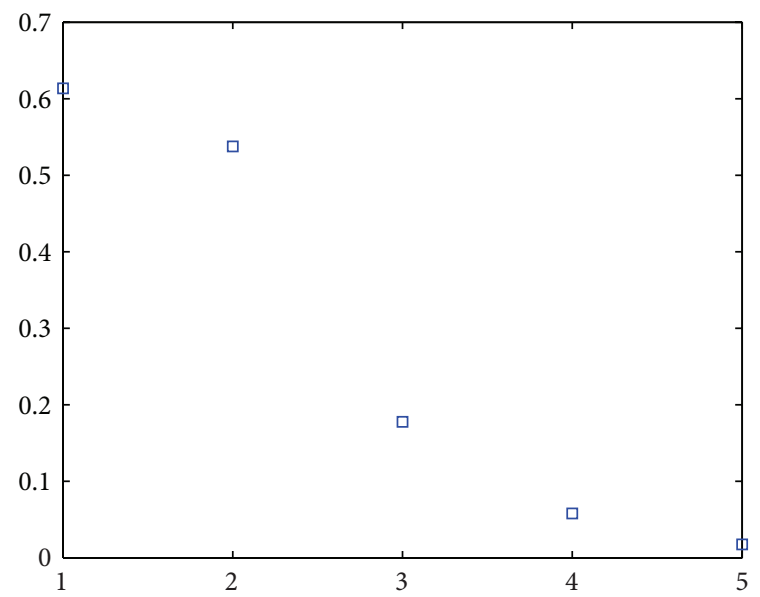

FIGURE 4: Error of two probabilities with ratio $<1$. 
TABLE 1: Experimental comparison for ant number $m$.

\begin{tabular}{lccccccc}
\hline$m$ & Best & Worst & Average & STD & $P_{A}$ & $P_{g}$ \\
\hline 2 & 2.6907 & 3.2025 & 2.7671 & 0.0840 & 0.8496 & 0.9985 & 0.1489 \\
4 & 2.6907 & 2.9689 & 2.7200 & 0.0521 & 0.9626 & 1.0000 & 0.0374 \\
5 & 2.6907 & 2.9689 & 2.7111 & 0.0410 & 0.9792 & 1.0000 \\
8 & 2.6907 & 2.8982 & 2.6966 & 0.0222 & 0.9972 & 1.0000 & 0.0208 \\
10 & 2.6907 & 2.8982 & 2.6937 & 0.0163 & 0.9992 & 1.0000 & 0.0028 \\
\hline
\end{tabular}

The best solution, the worst solution, the average solution quality, and the standard deviation in $K$ times running are given.

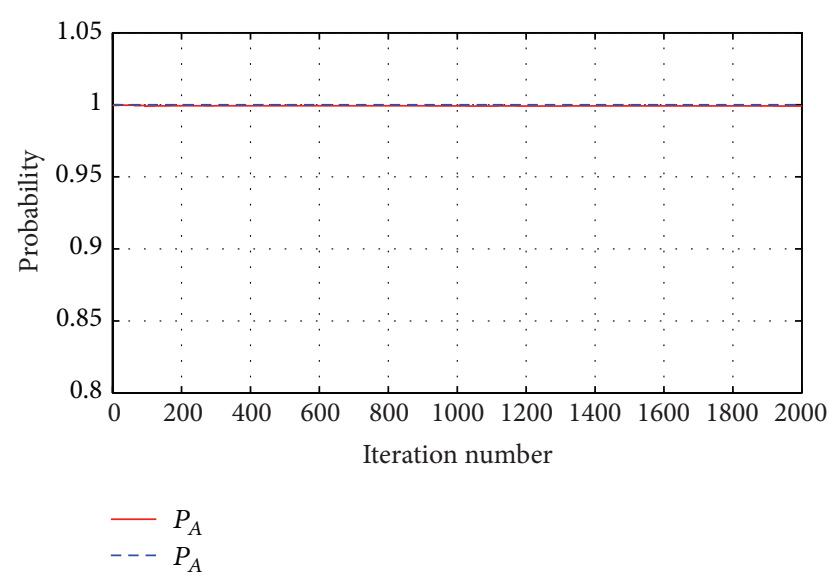

FIGURE 5: Comparison of two probabilities with ACO.

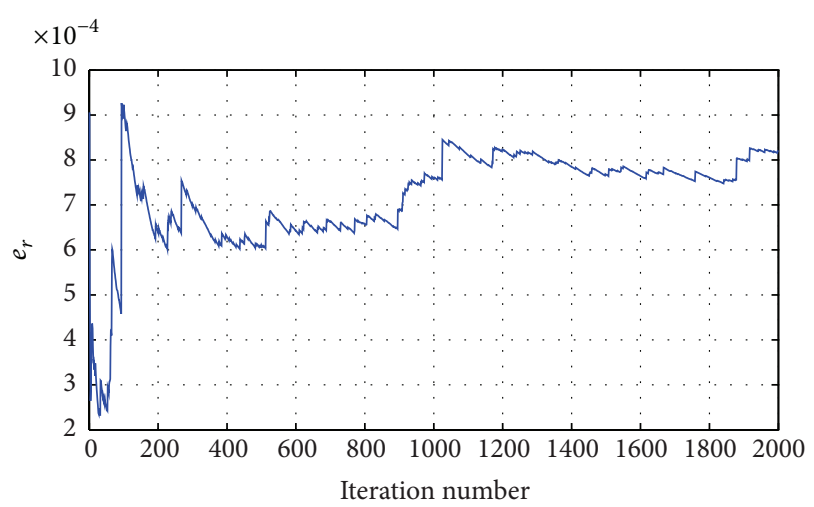

FIgURE 6: Error of two probabilities with ACO.

0.9. In Algorithm 1 and Figure 1, we can see that the error of two probabilities is high. But the error decreases with the ratio increasing.

4.3. Ordinal Performance Evaluation of ACO. Let $K=2000$; the computational results can be seen from Figures 5 and 6 . From Figure 5 we can see that the alignment probability of $P_{A}$ and $P_{g}$ is close to 1 and the difference is low. The $P_{A}$ is slightly lower than $P_{g}$. It is showed that the evaluation method is conservative. The error range is less than $0.1 \%$. This shows that the calculation result is credible.
In order to further study the relation between the parameters of ant colony algorithm and evaluation results, we focus on the relationship between the maximum number of iterations changes and ant number changes and evaluation of results. The results can be seen from Tables 1 and 2 .

First, we study the ant number. The ant number $m$ belongs to the set $\{2,4,5,8,10\}$. From Table 1 we can see that the value of $P_{A}$ is increasing with the $m$ increasing. The error of probability is reducing with the $m$ increasing. This shows that the size of solution has some influence on the evaluation method. Second, we study the iteration number $N_{\max }$ which is selected from the set $\{10,20,30,50,100,200\}$. From Table 2 we can see that $P_{A}$ is much less than $P_{g}$ when $N_{\max }$ is 10. But, with $N_{\max }$ increasing, the error is reducing. The reason is that the information of space is accumulated with $N_{\max }$ increasing. It is showed that the more the utilization of information of the solution space, the more accurate the result.

4.4. Ordinal Performance Evaluation of PSO and AFS. We also do the same comparison for PSO and AFS. The results can be seen from Tables 3 and $4 . m$ is particle number in Table 3 and $m$ is fish number in Table 4. From Tables 3 and 4 we can see that the value of $P_{A}$ is increasing with the $m$ increasing and the maximum number of iterations $N_{\max }$. The average solution is also improved. It is showed that the solution quality is effect on $P_{A}$.

\section{Conclusion}

A solution assessment method of SI is presented in this paper. Based on the analysis of the existing knowledge foundation, combined with the ordinal optimization theory, the ordinal performance is research target to evaluate solution. Then based on the analysis of characteristics of SI algorithms, the framework of evaluation method is given. The detailed steps of the method are presented. Finally, taking the Hopfield 10city problem as an example, some simulation experiments are done. The experimental results show that the proposed method is feasible.

\section{Conflict of Interests}

The authors declare that there is no conflict of interests regarding the publication of this paper. 
TABLE 2: Experimental comparison for maximum iteration number.

\begin{tabular}{lccccccc}
\hline$N_{\max }$ & Best & Worst & Average & STD & $P_{A}$ & $P_{g}$ \\
\hline 10 & 2.6907 & 3.1879 & 2.7695 & 0.0791 & 0.7750 & 0.9985 \\
20 & 2.6907 & 2.9844 & 2.7138 & 0.0435 & 0.9586 & 1.0000 & 0.2235 \\
30 & 2.6907 & 3.0504 & 2.7118 & 0.0440 & 0.9748 & 1.0000 \\
50 & 2.6907 & 2.9390 & 2.7094 & 0.0393 & 0.9803 & 1.0000 & 0.0252 \\
80 & 2.6907 & 2.9669 & 2.7073 & 0.0384 & 0.9835 & 0.0197 \\
100 & 2.6907 & 2.9669 & 2.7061 & 0.0358 & 0.9853 & 1.0000 \\
200 & 2.6907 & 2.8982 & 2.7022 & 0.0327 & 0.9909 & 1.0000 \\
\hline
\end{tabular}

TABLE 3: Experimental comparison for PSO.

\begin{tabular}{ccccccccc}
\hline$m$ & $N_{\max }$ & Best & Worst & Average & STD & $P_{A}$ & $P_{g}$ \\
\hline 10 & 30 & 2.6907 & 3.5976 & 3.0174 & 0.1543 & 0.6720 & 0.7425 & 0.0713 \\
10 & 50 & 2.6907 & 3.3618 & 2.9483 & 0.1310 & 0.7861 & 0.8890 & 0.1029 \\
10 & 60 & 2.6907 & 3.3582 & 2.9227 & 0.1222 & 0.8257 & 0.9340 \\
10 & 80 & 2.6907 & 3.3038 & 2.8923 & 0.1104 & 0.8894 & 0.9745 & 0.1083 \\
10 & 100 & 2.6907 & 3.2328 & 2.8685 & 0.1029 & 0.9262 & 0.9840 & 0.0578 \\
20 & 60 & 2.6907 & 3.2275 & 2.8516 & 0.0947 & 0.9480 & 0.9970 \\
20 & 100 & 2.6907 & 3.0861 & 2.8068 & 0.0736 & 0.9898 & 0.0490 \\
\hline
\end{tabular}

TABLE 4: Experimental comparison for AFS.

\begin{tabular}{lcccccccc}
\hline$m$ & $N_{\max }$ & Best & Worst & Average & STD & $P_{A}$ & $P_{g}$ & $e_{r}$ \\
\hline 5 & 50 & 2.6907 & 3.1556 & 2.7439 & 0.1017 & 0.7036 & 0.9985 & 0.2949 \\
8 & 50 & 2.6907 & 3.0909 & 2.7108 & 0.0569 & 0.8215 & 1.0000 & 0.1785 \\
10 & 50 & 2.6907 & 3.0783 & 2.7034 & 0.0440 & 0.8639 & 1.0000 & 0.1361 \\
15 & 50 & 2.6907 & 3.0302 & 2.6929 & 0.0153 & 0.9397 & 1.0000 & 0.0603 \\
10 & 80 & 2.6907 & 3.0344 & 2.6970 & 0.0293 & 0.8855 & 1.0000 & 0.1145 \\
10 & 100 & 2.6907 & 3.0830 & 2.6956 & 0.0280 & 0.8898 & 1.0000 \\
20 & 80 & 2.6907 & 2.7782 & 2.6909 & 0.0039 & 0.9743 & 1.0000 & 0.1102 \\
20 & 100 & 2.6907 & 2.7782 & 2.6909 & 0.0048 & 0.9750 & 1.0000 & 0.0257 \\
\hline
\end{tabular}

\section{Acknowledgments}

This work was supported by the National Natural Science Foundation of China (Grant nos. 61305149, 61104222, and 61304175) and the Science Fundamental Research Project of Jiangsu Normal University (Grant no. 12XL063). The authors are also thankful to the anonymous referees.

\section{References}

[1] J. Kennedy, R. C. Eberhart, and Y. H. Shi, Swarm Intelligence, Morgan Kaufmann, San Mateo, Calif, USA, 2001.

[2] M. Dorigo and T. Stützle, Ant Colony Optimization, MIT Press, Cambridge, Mass, USA, 2004.

[3] J. Kennedy and R. Eberhart, "Particle swarm optimization," in Proceedings of the IEEE International Conference on Neural Networks, pp. 1942-1948, December 1995.

[4] D. Karaboga and B. Akay, "A survey: algorithms simulating bee swarm intelligence," Artificial Intelligence Review, vol. 31, no. 14, pp. 61-85, 2009.

[5] X.-L. Li, Z.-J. Shao, and J.-X. Qian, "An optimizing method based on autonomous animals: fish-swarm algorithm," System Engineering Theory and Practice, vol. 22, no. 11, pp. 32-38, 2002.
[6] M. Dorigo, V. Maniezzo, and A. Colorni, "Ant system: optimization by a colony of cooperating agents," IEEE Transactions on Systems, Man, and Cybernetics, B: Cybernetics, vol. 26, no. 1, pp. $29-41,1996$.

[7] M. Dorigo and L. M. Gambardella, "Ant colony system: a cooperative learning approach to the traveling salesman problem," IEEE Transactions on Evolutionary Computation, vol. 1, no. 1, pp. 53-66, 1997.

[8] T. Stützle and H. H. Hoos, "MAX-MIN ant system," Future Generation Computer Systems, vol. 16, no. 8, pp. 889-914, 2000.

[9] Z. J. Zhang and Z. R. Feng, “Two-stage updating pheromone for invariant ant colony optimization algorithm," Expert Systems with Applications, vol. 39, no. 1, pp. 706-712, 2012.

[10] J. Aspnes and O. Waarts, "Compositional competitiveness for distributed algorithms," Journal of Algorithms, vol. 54, no. 2, pp. 127-151, 2005.

[11] J. Hsler, P. Cruz, A. Hall, and C. M. Fonseca, "On optimization and extreme value theory," Methodology and Computing in Applied Probability, vol. 5, no. 2, pp. 183-195, 2003.

[12] D. P. Bertsekas, Nonlinear Programming, Athena Scientific Press, Belmont, Mass, USA, 1999. 
[13] H. H. Hoos and T. Stützle, Stochastic Local Search: Foundations and Applications, Morgan Kaufmann Press, San Francisco, Calif, USA, 2005.

[14] M. A. Montes de Oca, T. Stützle, M. Birattari, and M. Dorigo, "Frankenstein's PSO: a composite particle swarm optimization algorithm," IEEE Transactions on Evolutionary Computation, vol. 13, no. 5, pp. 1120-1132, 2009.

[15] Y. C. Ho, R. S. Sreenivas, and P. Vakili, "Ordinal optimization of DEDS," Discrete Event Dynamic Systems, vol. 2, no. 1, pp. 61-88, 1992.

[16] Z. Shen, Y.-C. Ho, and Q.-C. Zhao, "Ordinal optimization and quantification of heuristic designs," Discrete Event Dynamic Systems: Theory and Applications, vol. 19, no. 3, pp. 317-345, 2009.

[17] Z. Shen, Q.-C. Zhao, and Q.-S. Jia, "Quantifying heuristics in the ordinal optimization framework," Discrete Event Dynamic Systems: Theory and Applications, vol. 20, no. 4, pp. 441-471, 2010. 

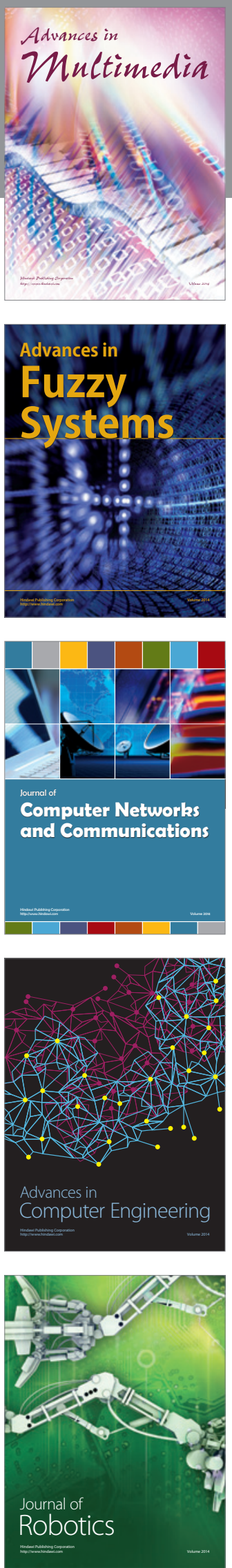

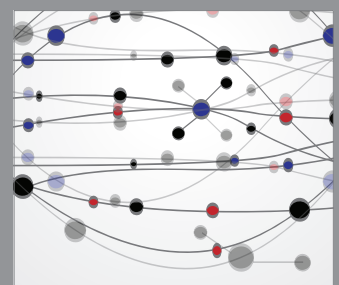

The Scientific World Journal
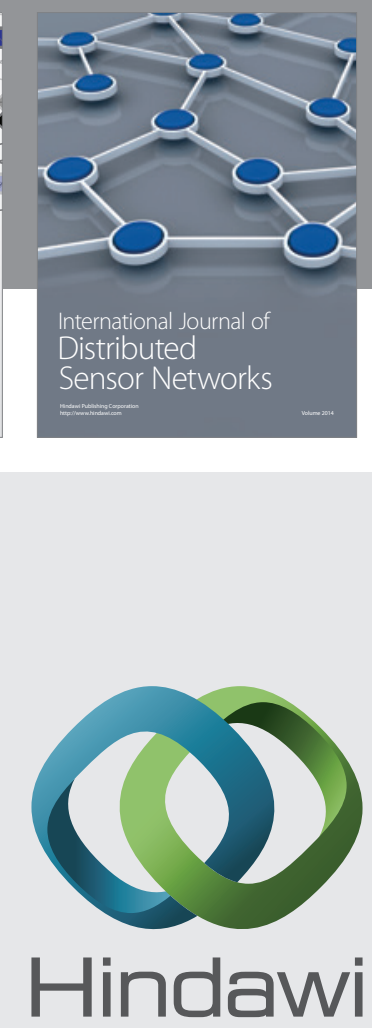

Submit your manuscripts at

http://www.hindawi.com
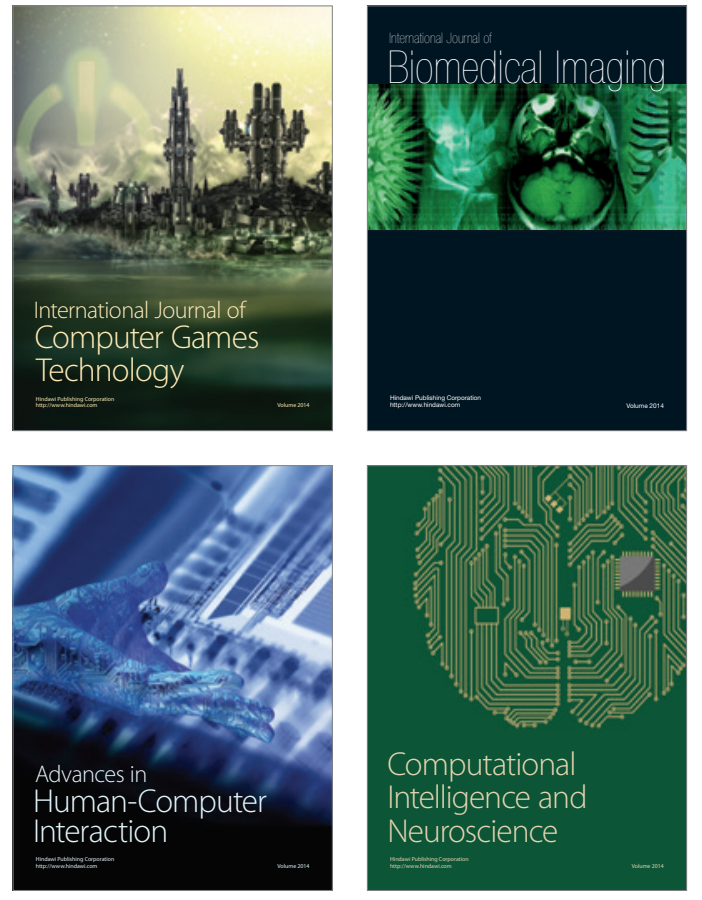
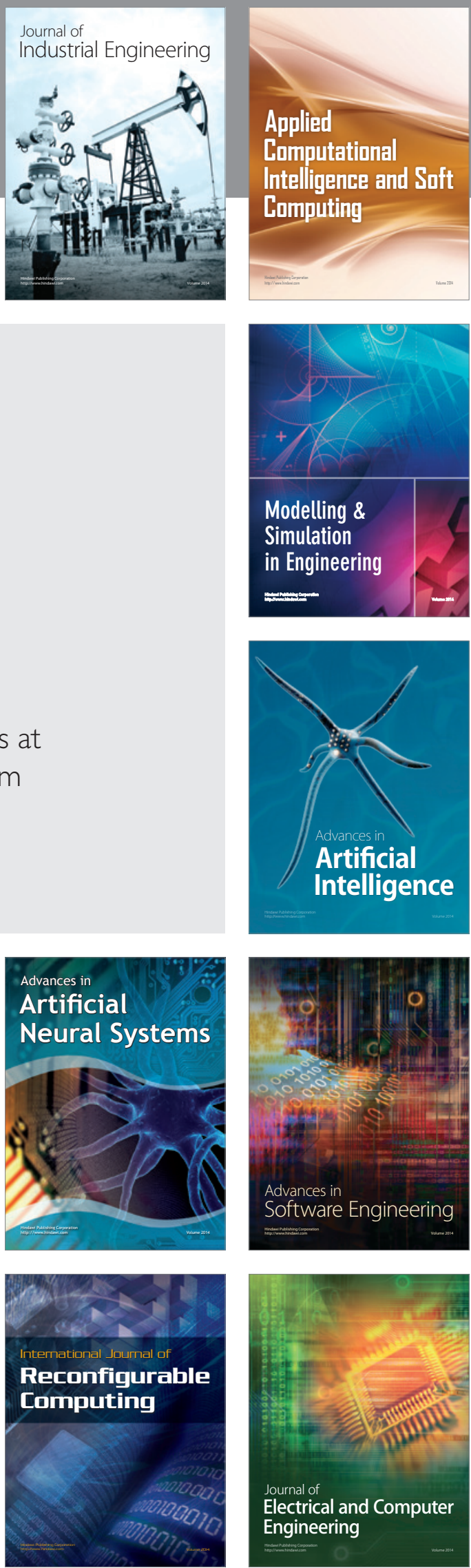